\title{
Optimal Decision-Making With Time Diversification
}

\author{
PAOLO VANINI and LUIGI VIGNOLA
}

\begin{abstract}
One of the most enduring topics in financial theory is the persistence of investment risk across time. Traditional finance lacks methods for considering and hedging non-diversifiable risks. This paper is based on the general equilibrium model of Allen and Gale (1997). We extend their model in various directions: the intermediary is a firm and not a planner, financial markets are assumed to be incomplete, and the mechanism of intergenerational risk-sharing is endogenously determined. Our model allows for the analysis of optimal behavior of individuals and the intermediary together with the respective feedback processes.
\end{abstract}

JEL-Classification: G10, G20, D91

Keywords: Cross-Sectional Risk, Intertemporal Risk-Smoothing, RiskSharing, Time Diversification and Incomplete Financial Markets

Acknowledgments: We are indebted to Douglas Gale and Simon Benninga (the editor) for their comments and suggestions. We would also like to thank Martin Janssen, Kurt Ris, Fabio Trojani and the participants of various seminars at the Universities of Zurich and Lugano, Switzerland for fruitful discussions, and Claudia Boon for preparing the manuscript for publication. All errors are solely the authors' responsibility. Financial support from ECOFIN Research and Consulting AG, Zurich, Switzerland, is gratefully acknowledged. We also thank our current employer, Zürcher Kantonalbank, for making part of this research possible.

Address: Paolo Vanini (corresponding author), Zürcher Kantonalbank, Neue Hard 9, CH8010 Zurich, Switzerland, paolo . vanini a zkb . ch. Luigi Vignola, Zürcher Kantonalbank, Uraniastrasse 31+35, CH-8001 Zurich, Switzerland, luigi . vignola@ zkb. ch. 


\section{Introduction}

The "oil shock" in the 1970s had a dramatic effect on the value of firms. Investors forced to liquidate portfolios representing a significant portion of their assets at lower than expected stock prices had to reduce their consumption for the rest of their lives. Davis (1995) reports, for example, that Britons who retired in 1974 and had contribution-based pensions without a minimum guarantee received an income for the remainder of their lives which was worth only half that of individuals who retired before the 1973 shock. In countries such as Germany, where savings are mostly held with intermediaries (banks, insurance companies, pension funds), individuals did not lose as much wealth as their counterparts in the US or the UK. In the 1980s the situation was reversed due to booming stock markets.

The effect of the oil shock on financial markets is considered to be a non-diversifiable risk. The example suggests, however, that the timing of such risks can be crucial for individuals. We call these risks in individuals' optimal planning longitudinal risks. Traditional finance lacks methods for reducing or hedging such risks. Instead, theory focuses on the efficient sharing of risks through exchange (cross-sectional risk sharing). To show the importance of non-diversifiable risk for individuals, let us consider the following exercise.

\section{Insert Figure 1 about here}

We assume that individuals start saving 20 years before they retire. To simplify matters, let us say they invest their wealth either in a risk-free asset with 5 percent return per year or in a risky asset based on all assets on the NYSE, Nasdaq and AMEX. Figure 1 shows the average growth rate of wealth for each generation up to the time of retirement. Two major observations are immediate. Due to booming stock markets in the 90s, individuals who started investing in the stock market twenty years before will outperform by a wide margin those individuals who invested in risk-free assets in the same period. By contrast, most individuals who retired in the 70s and had invested in stocks - although broadly diversified - underperformed the risk-free investment.

It is often argued that for increasing time horizons, the investment danger shown in Figure 1 vanishes. For this purpose, let us consider individuals with an 18,20, 22 and 24-year investment horizon before retirement. Again. from Figure 1, we see that wealth at retirement is by no means proportional to the investment periods. In other words, even for generations that are very close to each other, wealth on retirement depends on a risk factor which cannot be diversified using common stocks or derivatives on stocks. This example illustrates that longitudinal risks are crucial for individuals. Furthermore, any theoretical solution to this problem cannot be based on "averages" (e.g. average wealth distribution and average over the 
duration of the saving period) since, as shown in Figure 2, any solution based on "averages" is not satisfactory for the agents.

\section{Insert Figure 2 about here}

In this paper we expand upon the traditional approach by considering optimal decision-making on the part of individuals and intermediaries where cross-sectional and longitudinal risk sharing is allowed. Two types of agents are considered: The first agent is an individual who maximizes expected utility within a finite time horizon, while at the same time financing her optimal consumption path. The second agent is an intermediary who maximizes expected utility within an infinite time horizon, while at the same time offering intertemporal smoothing of consumption and incentivecompatible risk-sharing contracts to the first type of agents.

Risks which cannot be diversified away at a given point in time, can nevertheless be averaged over time in a way that reduces their impact on individual welfare. One could think of a complicated contracting system between individuals of the same generation and between individuals of different generations, where some crucial points in time (such as retirement) are at a different distance in time for different individuals. Bodie et al. (1992) argue, for example, that younger workers should bias their investment towards equities on the grounds that - unlike older workers - they can offset equity losses by working harder later. Although this complicated intra- and inter-generational longitudinal risk-sharing contract may be efficient, the inherent asymmetric information problems make such a solution of the non-diversifiable risk problem highly implausible. A more convincing mental exercise is the introduction of a new player: an intermediary with the promise to redistribute the longitudinal risks between the individuals in the form of bilateral contracts.

One of the characteristic features of the intermediary is that a sudden fall in asset values does not imply losses that cannot be offset over time. Two provisions are sufficient to achieve this goal: a persistent income from the individuals and a buffer stock that is used as a capital cushion in order to absorb the impact of longitudinal risk.

A possible design of the provision is given by bilateral risk-sharing contracts with the individuals of each generation. Allen and Gale (1997) present a two-period overlapping generation model (OLG) and prove the existence of a stationary Markov equilibrium in which the price of the risky asset is constant and the demand for the risk-free asset is zero; i.e., in equilibrium the risk-free asset is not necessary in order to hedge against the uncertainty of the risky asset's return. Within their parameters, they show the existence of feasible allocations that almost entirely eliminate risk in the long run. In summary, Allen and Gale (1997) first prove that market incompleteness leads to the absence of intertemporal smoothing and then show how a financial system with intermediaries can indeed eliminate the 
resulting inefficiencies. In their model the OLG structure is a metaphor for the incomplete market; the preferences of the intermediary defining intertemporal smoothing are just averaged individual preferences, the optimal risk-sharing contracts are not determined, and the interplay between crosssectional diversification and intertemporal smoothing is not analyzed. Our model addresses some of these issues. It is based on the following extensions:

- A truly incomplete financial market is considered. In addition to the risk-free asset there are two risky assets. In consequence, the interplay between cross-sectional risk sharing and intertemporal smoothing can be analyzed. Both types of agents - intermediary and individuals - are in the same financial market and arbitrage is excluded.

- The intermediary is a firm with its own preference structure. The optimization of expected utility is done under the condition of intertemporal smoothing, budget constraint, and the risk-sharing contract participation constraint for the individuals.

- The risk-sharing contracts are agreements between the intermediary and representative agent of each generation. Hence, there is an infinite number of individuals in our model who choose their best policy: agents, born at each time $\mathrm{n}$ and living for a finite number of years, and the intermediary, which lives for ever.

Aside from these extensions, there is also an important restriction compared to Allen and Gale (1997): We work in a partial equilibrium framework. This framework allows for the analysis of the following problems:

- Optimal individual behavior: What is the impact of varying initial wealth of the individuals on the acceptance of the offered risksharing contract? How does a variation in the time horizon affect the willingness of the individuals to accept the risk-sharing contract? How does risk sharing affect the cross-sectional diversification? Under what conditions can market incompleteness be overcome by an agent's self-insurance possibilities?

- Optimal behavior of the intermediary: What are the optimal risksharing conditions, and what is a reasonable model of intertemporal smoothing of consumption?

- Application to pension fund economics: What is the impact of voluntary risk-sharing compared to its compulsory counterpart? Is there a rationale for the existence of security funds and "fund-offund" systems?

The paper is organized as follows. In Section Two we present the model and compare it with the model of Allen and Gale (1997). In Section Three the individual behavior is analyzed. Section Four considers the optimal behavior of the intermediary, and in Section Five we discuss the our results. The proofs of the propositions are given in appendices. 


\section{The Model}

Our model focuses on some aspects of the overlapping generation model of Allen and Gale (1997). Contrary to their assumption, in our model the agents act as price takers. Therefore, only a partial equilibrium will be analyzed. This simplification allows us to enrich their model in the following way: the risk-sharing mechanism is modeled and a primary goal is to determine the optimal contracts in an imperfect financial market.

We first describe our model and at the end of the section discuss its relationship to the model to Allen and Gale (1997).

The OLG model serves as a vehicle for the analysis of intertemporal risk smoothing. Time is divided into a finite number of dates $n=1,2, \ldots$, and a new generation is born at each date $n$. Since we assume that each generation consists of an equal number of identical agents, there is no loss of generality in considering a representative agent of each generation. Initially, there is one old generation that lives for just one period; each subsequent generation lives for two periods. We rule out the possibility that the agents can self insure themselves (cf. Schechtman [1976]). The agents can consume a single good $c_{n}$ in period $n$, and agents born at the beginning of period $n$ have an endowment $W_{n}$ but no endowment in $n+1$. This mimics the life cycle.

A second agent, the intermediary, lives forever. The intermediary maximizes its utility function under a number of constraints to be described below. Before proceeding with the definition of the model for different agents, we first consider the structure of the financial market. There are two types of general tradeable assets, a safe asset $B_{n}$ with a constant, deterministic interest rate $r$, and two risky assets $S_{n}^{(1)}$ and $S_{n}^{(2)}$. We use two risky assets, since one of our goals is to analyze cross-sectional (diversifiable) risk in addition to intertemporal smoothing of risk (non-diversifiable risk). We assume the existence of four states of the world $\omega_{k}$. Hence, the payoff-matrix for the agent of generation $n$ reads:

$$
H_{n+1}=\left(\begin{array}{ccc}
B_{n+1}\left(\omega_{1}\right) & S_{n+1}^{(1)}\left(\omega_{1}\right) & S_{n+1}^{(2)}\left(\omega_{1}\right) \\
B_{n+1}\left(\omega_{2}\right) & S_{n+1}^{(1)}\left(\omega_{2}\right) & S_{n+1}^{(2)}\left(\omega_{2}\right) \\
B_{n+1}\left(\omega_{3}\right) & S_{n+1}^{(1)}\left(\omega_{3}\right) & S_{n+1}^{(2)}\left(\omega_{3}\right) \\
B_{n+1}\left(\omega_{4}\right) & S_{n+1}^{(1)}\left(\omega_{4}\right) & S_{n+1}^{(2)}\left(\omega_{4}\right)
\end{array}\right) .
$$

The objective probability $P$ is strictly positive, i.e., $P(\omega)>0 \forall \omega \in \Omega$.

ASSUMPTION 1. We assume that there are no arbitrage opportunities and that the financial market is incomplete. The set of risk neutral probability measures $\mathcal{Q}$ is assumed to be one-dimensional.

Without loss of generality, we consider two linear independent vectors $Q_{1}$ and $Q_{2}$ in the closure $\overline{\mathcal{Q}}$ of $\mathcal{Q}$, such that each element of $\mathcal{Q}$ can be written as a linear combination of these two vectors, where the weights (coordinates) can be arbitrary, but have to add up to one. 
In addition to these three securities, there is a fourth security consisting of a pair

$$
\left(f_{n}, T_{n+1}\right)^{\prime} \equiv\left(f_{n}, T\left(S_{n+1}^{(1)}, S_{n+1}^{(2)}\right)\right)^{\prime} \in \mathbb{R}^{2} .
$$

The first component is a deterministic payment from the young agent of generation $n$ to the intermediary, where $T_{n+1}$ is a contingent payment from the intermediary to the old agent of generation $n$ at time $n+1$. This security reflects a contract between two parties, where the young transfers wealth to the intermediary for a contingent transfer at a later point in time.

ASSUMPTION 2. We assume that the intermediary holds the bargaining power, i.e., the contract $\left(f_{n}, T_{n+1}\right)^{\prime}$ is a take-it or leave-it contract open to all individuals.

The contingent component of the contract influences the financial market structure as follows:

ASSUMPTION 3. The augmented payoff matrix $\tilde{H}_{n}$, which is the matrix $H_{n}$ augmented by the vector $T_{n}$, is of full rank for all $n \in \mathbb{N}$.

Therefore, there are two reasons why such a transfer is beneficial to an agent. First, the contract completes the financial market for the agent living in $n$, i.e., all contingent claims are marketable and cross-sectional risk can be eliminated. Second, with a "guarantee" from the financial intermediary (the exact meaning will be discussed below), the contract can eliminate nondiversifiable risk too, i.e., intertemporal risk smoothing across generations is achieved.

$V\left(W_{n}, n,\left(f_{n}, T_{n+1}\right)^{\prime}\right)$ denotes the value function of the individual born on date $n$ with initial wealth $W_{n}$ and a financial contract $\left(f_{n}, T_{n+1}\right)^{\prime} . J\left(A_{0}\right)$ is the value function of the intermediary with initial capital $A_{0}$. The utility functions $u$ of the individuals and $v$ of the intermediary are assumed to be twice continuously differentiable, increasing and strictly concave. Furthermore, the Inada condition $\lim _{c \rightarrow 0} u^{\prime}(c)=+\infty$ holds for the individuals, implying that the constraint $c \geq 0$ is never binding. The intermediary solves the problem (see Figure 2 for illustration and the appendices for a definition of the presented formulae)

$$
J\left(A_{0}\right)=\max _{\psi, y, f, T} \mathbb{E}\left[\sum_{n=0}^{\infty} \beta^{n} v\left(y_{n}\right)\right]
$$

subject to

$$
\begin{aligned}
& A_{n}=A_{n-1}+\left\langle\psi_{n-1}, \Delta S_{n}\right\rangle+f_{n}-T_{n}-y_{n}, A_{0} \text { given }, A_{n} \geq 0 \\
& \text { (ISC) } \eta \geq\left|\sum_{n \in \Lambda_{1}} V\left(W_{n}, n,\left(f_{n}, T_{n+1}\right)^{\prime}\right)-\sum_{m \in \Lambda_{2}} V\left(W_{m}, m,\left(f_{m}, T_{m+1}\right)^{\prime}\right)\right| \\
& \forall \Lambda_{1}, \Lambda_{2} \subset N:\left|\Lambda_{1}\right|=\left|\Lambda_{2}\right| \\
& \text { (PC) } \quad V\left(W_{n}, n,\left(f_{n}, T_{n+1}\right)^{\prime}\right) \geq V\left(W_{n}, n,(0,0)^{\prime}\right), \quad \forall n \in \mathbb{N}
\end{aligned}
$$


where $\Delta S_{n}=\left(B_{n}-B_{n-1}, S_{n}^{(1)}-S_{n-1}^{(1)}, S_{n}^{(2)}-S_{n-1}^{(2)}\right)^{\prime},\langle x, y\rangle$ denotes the scalar product of the vectors $x$ and $y$ and $\beta$ is the rate of time preference. The investment strategies $\psi_{n}=\left(\psi_{n}, \psi_{n}^{(1)}, \psi_{n}^{(2)}\right)^{\prime} \in \mathbb{R}^{3}$ denote the investment in the risk-free asset and the two risky assets, where short selling for the intermediary is allowed.

The optimization problem implies that the intermediary maximizes its lifetime utility by choosing strategies $\psi_{n}$, contract prices $f_{n}$, contingent payments $T_{n+1}$, and $y_{n}$ in an optimal way. This optimization is done under various constraints: the budget constraint, the intertemporal smoothing constraint (ISC), and the participation constraint (PC). The form of the budget constraint implies that only self-financing strategies are feasible. The participation constraint assures that each individual is better off accepting the contract proposed by the intermediary than choosing to invest solely in the incomplete financial market. Hence, (PC) for agent $n$ corresponds to the optimization problem

(PC)

$$
\begin{aligned}
V\left(W_{n}, n,\left(f_{n}, T_{n+1}\right)^{\prime}\right) & =\max _{c_{n}, \phi_{n} \in \mathcal{X}_{c o n, n}}\left\{u\left(c_{n}-\bar{c}_{n}\right)+\beta \mathbb{E}\left[u\left(c_{n+1}-\bar{c}_{n+1}\right) \mid \mathcal{F}_{n}\right]\right\} \\
& \geq \max _{\tilde{c}_{n}, \tilde{\phi}_{n} \in \mathcal{X}_{n}}\left\{u\left(\tilde{c}_{n}-\tilde{\bar{c}}_{n}\right)+\beta \mathbb{E}\left[u\left(\tilde{c}_{n+1}-\tilde{\bar{c}}_{n+1}\right) \mid \mathcal{F}_{n}\right]\right\} \\
& =V\left(W_{n}, n,(0,0)^{\prime}\right)
\end{aligned}
$$

where $\phi_{n}=\left(\phi_{n}, \phi_{n}^{(1)}, \phi_{n}^{(2)}\right)^{\prime} \in \mathbb{R}^{3,+}$ denotes the investment in the various financial assets and $\left\{\mathcal{F}_{n}\right\}_{n \in N}$ the filtration assumed to be generated by the prices of the risky assets. The reference level path $\bar{c}_{n}$ is exogenously given, reflecting the basic finding that humans are often more sensitive to how an outcome differs from some reference outcome than to the absolute value of the outcome itself. The constraint set $\mathcal{X}_{c o n, n}$ of $c_{n}, c_{n+1}$ and the investment strategy $\phi_{n}$ is such that the budget constraints hold

$$
\begin{aligned}
c_{n}+\left\langle\phi_{n}, S_{n}\right\rangle & =W_{n}-f_{n} \\
T_{n+1}+\left\langle\phi_{n+1}, S_{n+1}\right\rangle & =c_{n+1} .
\end{aligned}
$$

The conditions defining $\mathcal{X}_{n}$ are similar to the ones given in (PC).

With (PC) the intermediary guarantees the individual's participation. The criterion for participation - (conditional) expected utility - is, however, not adequate for the consideration of intertemporal smoothing. Therefore (ISC) is introduced as an additional condition in the intermediary's optimization problem. This condition compares the value functions of two sets of generations living at different calendar dates and ensures that the sum of the values of the two sets of generations cannot fluctuate in an arbitrary way: These fluctuations are bounded by a constant $\eta$. A short mental exercise is in order. Let us assume two sets of generations, $A$ and $B$, separated in time, and that the financial market performs poorly during the lifetime 
of $B$. Without the intermediary, individuals in $B$ would be unable to consume as much as their counterparts in $A$. Therefore, the sum of their value functions would be smaller than for those in $A$. With the presence of an intermediary and (ISC), the discrepancies cannot exceed the value $\eta$. This can be achieved by choosing the risk-sharing contracts for generations $A$ and $B$ appropriately, i.e. the generations in $B$, will be able to consume more than they would without the intermediary. The extra resources provided to them by the intermediary are taken from the buffer stock which had previously been accumulated by the individuals in $A$. This is established by the assumption of a net inflow for the intermediary due to the absence of shocks. Clearly, if stocks are underperforming all the time, intertemporal smoothing of consumption by an intermediary is not feasible. Therefore, an initial critical phase during which the buffer stock is accumulated has to be mastered first. Condition (ISC) thus separates the performance of an individual born at a specific date from the financial market fluctuations during his life. In other words, the decisions of the individual are time invariant, which is the essence of removing longitudinal risk.

We refer the interested reader to the book by Hernandez and Lassere (1996) for necessary and sufficient conditions for a solution of the problem presented above. Basically, the problem can be reduced to the compactness of the feasible set of decisions. Given the compactness of the feasible set and some other technical conditions, the crucial measurable selection conditions hold (cf. Hernandez and Lassere [1996], Chapter III), and can be solved with the aid of the Bellman equation (at least theoretically). Since we are interested in explicit expressions, the general functions need to be approximated. We restrict our analysis to first-order approximations, since the expressions become rather involved in second-order approximations. Hence, if, for example, the utility function of individuals is approximated, only marginal utility matters for the result, and the curve of the utility function is ignored. In order to carry out the analysis, we assume that all functions are real-analytic, i.e., that they possess a Taylor series expansion which represents the function.

Clearly, if we do the approximations and solve the resulting model, the solution is not that of the original model. But under suitable technical conditions, the original model has a solution (cf. Hernandez and Lassere [1996], Chapter III), and the same holds true for each approximation model to all orders in a Taylor series expansion. Hence, we have a solution $s^{*}$ of the original model and a sequence $\left(s_{n}\right), n=1, \ldots, N$, of solutions of the approximation models. First, one has to show that the sequence converges. We can use the so-called policy iteration procedure for this. Second, using successive approximations and a properly defined measurable selection condition, one can show that the limit of the sequence is equal to $s^{*}$. Since a detailed analysis of these technical issues is beyond the scope of this paper, we leave it for future research. 
How is our model related to the model of Allen and Gale (1997)? Most importantly, their model is an equilibrium model, while in our partial equilibrium model the equilibrium is the solution of the optimization problem outlined above, since there are no prices. The decision to work in a partial equilibrium framework is due to the authors' focus to analyze and characterize as explicitly as possible a solution of the "time-diversification" problem. Hence, the goal is not to derive a rationale as Allen and Gale did for the existence of an intermediary, but to assume the equilibrium properties and investigate upon the properties of the partial equilibrium. The loss of generality is compensated for by the possibility of characterizing more explicitly than in the general setup the behavior of agents and of the intermediary.

The basic conditions, which we claim are a reasonable approximate of the basic characteristics of the equilibrium model are (ISC) and (PC). The former, which captures the intuition of intertemporal smoothing that expected utilities of different generations are not "too different" from one another, requires some explanation. So far, nothing has been said about (i) why it is rational for an intermediary to use such a constraint in the optimization program, and (ii) whether the proposed formulation of the intertemporal smoothing condition is a natural one. To answer the first question, let us look at Figure 4. It shows the optimality conditions for the intermediary and the agents: The risk-sharing contract is the chosen variable of the intermediary, and the agents choose consumption. It follows from Proposition 5 and (A.3) that if no (ISC) is considered, the optimality ratios $\frac{\partial v}{\partial v_{f_{n}}} / \frac{\partial v}{\partial v_{T_{n+1}}}$ and $\frac{\partial u}{\partial v_{c_{n}}} / \frac{\partial v}{\partial v_{c_{n+1}}}$ are the same (point $A$ ) on the optimal contracting line $h$ (this also follows from Proposition 5 and (A.2) and (A.2)). Therefore, in the case where only participation constraints are considered, the intermediary cannot be distinguished from the agents. By contrast, if (ISC) holds, the intermediary's optimal contract offered to the agent is at time $\mathrm{n}$ generically either on the line $g$ (decreasing buffer stock) or on the line $f$ (increasing buffer stock, see again Proposition 5 and the following discussion). Hence, in case $g$ the intermediary is better off if (ISC) does not bind, but is worse off in case $f$. Hence, the intermediary is indifferent to whether (ISC) is used in the program or not. At this point, an expectation about future moves in market prices matter. This also demonstrates the limitations of intermediaries as redistributors of wealth between different generations. If one expects that the average return on assets is positive in the long run, the intermediary will be able to choose contracts on the line $g$ most of the time. Therefore, on average it is rational to use the (ISC) condition. If on the contrary, average returns on risky assets are supposed to be negative (more precisely: lower than the return on the risk-free asset), it does not pay for an intermediary to offer intertemporal smoothing. This also highlights when the danger of default of intermediaries is greatest (pension funds for example): a long period where risky asset returns are low or even negative. 


\section{Insert Figure 3 about here}

The form of the ISC condition is by no means a unique one, but it is a rather general condition, and as we see below, it turns the qualitative equilibrium results of Allen and Gale (1997) into intuitive quantitative results. Instead of working with the value function of the agents, one could also state the smoothing objective directly in terms of the agent's consumption. This simplifies some calculations, but the agents are considered risk neutral if the intermediary chooses the optimal contracts. If the individual's value function is replaced by averages, the problem also becomes much simpler. But in this case, we basically do not remove longitudinal risk, since an averaging criterion as shown in the introduction can be disastrous.

In summary, it is rational for the intermediary to use (ISC) if on average the risky asset price returns are expected to be positive in the long run. This intuition captures the equilibrium results of Allen and Gale (1997). There, an initial phase of positive price returns is also needed to set up the buffer stock. Otherwise, the existence of an intermediary does not remove any inefficiencies due the incomplete financial market.

\section{Optimal Behavior of the Individuals}

We first analyze the individual's participation constraints. To simplify the notation we use $\mathbb{E}_{n}[\cdot]$ to denote the conditional expectation $\mathbb{E}\left[\cdot \mid \mathcal{F}_{n}\right]$.

Proposition 4. The individual born at time $n$ accepts the contract $\left(f_{n}, T_{n+1}\right)^{\prime}$ up to first-order (i.e., up to o $\left.\left(\left|c_{n}\right|\right), o\left(\left|c_{n+1}\right|\right)\right)$ if, and only if,

$$
c_{n}-\bar{c}_{n} \geq \tilde{\beta}_{n} \mathbb{E}_{n}\left[c_{n+1}-\bar{c}_{n+1}\right],
$$

where

$$
\tilde{\beta}_{n}:=\beta \frac{u^{\prime}\left(\bar{c}_{n+1}\right)}{u^{\prime}\left(\bar{c}_{n}\right)}
$$

and o denotes the Landau symbol. The consumption sequence $\left(c_{n}\right)$ is optimal if the offered contract is accepted, whereas $\left(\bar{c}_{n}\right)$ corresponds to the optimal consumption sequence without risk-sharing contracts.

Proof. Appendix A.

The participation constraint (PC) can be interpreted as follows. Consider an agent $A$ who is poor when young and who has a large quantity of highly non-tradable human capital. It is evident that tomorrow the individual expects to have transformed his human capital into income in such a way that the consumption $\bar{c}_{n+1}>\bar{c}_{n}$ exceeds today's possible consumption. By contrast, let $B$ be a satiated individual with consumption level $\bar{c}$, i.e., we interpret $B$ as a rich individual and $\bar{c}_{n+1} \sim \bar{c}_{n}$. In all other respects, individual $B$ is identical to $A$. (PC) then implies that individual $A$ is more likely to accept a contract than individual $B$. 
A natural generalization of the individual's situation outlined above is to consider agents living for more than two periods. This situation introduces the possibility of self-insurance of the agents over time. Schechtman (1976) proved that by considering large time horizons individuals may be able to considerably reduce risk on their own. In our model we are able to consider how the two different effects - self-insurance and risk sharing with an intermediary - interact. The model for the intermediary remains the same as in Section 2, but the agent born at time $n$ maximizes the expected utility

$$
\mathbb{E}_{n}\left[\sum_{j=n}^{n+M} \beta^{j} u\left(c_{j}\right)\right]
$$

if the agents live for $M$ periods. Using the martingale approach, we obtain for the budget constraint the case in which it pays to accept the contract

$$
\mathbb{E}_{Q, n}\left[\sum_{j=n}^{n+M} \frac{u\left(c_{j}\right)}{B_{j}}-\frac{T_{k}}{B_{k}}\right]=W_{n}-f_{n}, \quad n<k \leq n+M,
$$

with $Q$ being the risk-neutral probability (the market is complete in this case). Equation (PC) can be interpreted in a way such that the agent pays a fee $f_{n}$ at time $n$ against a stochastic payoff $T_{k}$ at time $k$, which may be realized before $M+n$.

The contracting possibilities remain the same, but the possibility of selfinsurance is introduced into the model. In the agent's optimization, if it does not pay to accept the contract, the individual adopts the same objective function (PC), but the budget constraint is replaced by a number of restrictions equal to the number of probability measures spanning the set of risk neutral probability measures. A necessary condition for the agent to accept a contract (up to the first-order in consumption) is

$$
c_{n}-\bar{c}_{n} \geq \kappa_{n, M}^{m}(\beta) \mathbb{E}_{n}\left[c_{m}-\bar{c}_{m}\right] \quad m>n,
$$

where

$$
\kappa_{n, M}^{m}(\beta):=(M-1) \beta^{n-1}(1-\beta)^{M-n} \frac{u^{\prime}\left(\bar{c}_{m}\right)}{u^{\prime}\left(\bar{c}_{n}\right)}
$$

and $m$ is the minimum time period such that

$$
c_{m}-\bar{c}_{m} \leq c_{k}-\bar{c}_{k} \quad \forall k \in\{n+1, \ldots, n+M\} .
$$

For $M \rightarrow \infty$, the incentive to share risks with the intermediary vanishes. This re-expresses the finding of Schechtman (1976) and the intuition that if agents live infinitely long, non-diversifiable risk due to market incompleteness can be overcome by the agent's self-insurance possibilities. This also fits the research called "time diversification" (cf. Jorion [1999]) which considers the impact of a varying time horizon on optimal decision-making. If $n \rightarrow M$, i.e. the decision time comes closer and closer to the final decision, the right-hand side of (PC) converges to zero, i.e., the contract will be 
accepted by any agent. This captures the intuition that it is difficult to selfinsure against a global drop in risky security prices if no time for recovery is left.

\section{Insert Figure 4 about here}

In Figure 4 consumption today $c_{n}$ and consumption tomorrow $c_{n+1}$ of an individual born at time $n$ are displayed on the axis. The four regions $A, B, C, D$ represent different investments in the two risky assets $S_{n}^{(1)}$ and $S_{n}^{(2)}$ of the individual if he accepts the offered risk-sharing contract relative to the case where there is no such contract (i.e., the financial market is incomplete). The magnitude of the optimal consumption depends on (i) the individual's preferences and (ii) initial wealth. Suppose that the preferences are the same for all individuals. Region $A$ can then be classified as the poor and $C$ as the rich individuals. As shown in the appendix, in region $A$ the individual will invest more in both risky assets if an intermediary exists compared to the case without an intermediary. On the other hand, in region $C$ there will be less investment in both risky assets compared to the case without the possibility of sharing risks with other generations through the intermediary. In regions $B$ and $D$, more is invested in one risky asset and less in the other one.

Therefore, if preferences are the same for all individuals and an intermediary exists, it is optimal for the poor to have a larger risk exposure than they would without an intermediary. For the rich, by contrast, it pays to invest more in the risk-free asset than in the case of an imperfect financial market. This shows that the statement "intermediaries allow the investors to bear more risk" should be used with caution, since the extent of the risk exposure depends crucially on preferences and the initial wealth of the individuals.

\section{Optimal Behavior of the Intermediary}

In the last section we analyzed the individual participation constraint of accepting a risk-sharing contract offered by the intermediary. Using the above results, we solve the problem of the intermediary in this section. The problem reads

$$
J\left(A_{0}\right)=\max _{\psi, y, f, T} \mathbb{E}\left[\sum_{n=0}^{\infty} \beta^{n} v\left(y_{n}\right)\right]
$$

subject to

$$
\begin{aligned}
A_{n} & =A_{n-1}+\left\langle\psi_{n-1}, \Delta S_{n}\right\rangle+f_{n}-T_{n}-y_{n}, A_{0} \text { given, } A_{n} \geq 0 \\
\text { (ISC) } \eta & \geq\left|\sum_{n \in \Lambda_{1}} V\left(W_{n}, n,\left(f_{n}, T_{n+1}\right)^{\prime}\right)-\sum_{m \in \Lambda_{2}} V\left(W_{m}, m,\left(f_{m}, T_{m+1}\right)^{\prime}\right)\right|
\end{aligned}
$$


$\forall \Lambda_{1}, \Lambda_{2} \subset N:\left|\Lambda_{1}\right|=\left|\Lambda_{2}\right|$,

(foPC) $\quad \frac{\gamma_{n}}{e} \mathbb{E}_{Q, n}\left[\frac{T_{n+1}}{B_{n+1}}\right] \geq \frac{\gamma_{n}}{e} f_{n}-\zeta_{n}+\tilde{\beta}_{n} \mathbb{E}_{Q, n}\left[\xi_{n}\right]$,

where

$$
\begin{aligned}
& \gamma_{n}=I^{\prime}(\bar{\lambda})\left(\frac{d_{0}}{e}\right)-\tilde{\beta}_{n} \mathbb{E}_{Q, n}\left[I^{\prime}\left(\frac{\alpha_{n} d_{0}}{e}\right) \alpha_{n}\right], \\
& \zeta_{n}=I\left(\frac{d_{0}}{e}\right)-I^{\prime}(\bar{\lambda})\left(\frac{d_{0}}{e}\right) \frac{d_{0}}{e}-I\left(\lambda_{1}+\lambda_{2}\right) \\
& \xi_{n}=I(\bar{\lambda})\left(\alpha_{n} \frac{d_{0}}{e}\right)-I^{\prime}(\bar{\lambda})\left(\alpha_{n} \frac{d_{0}}{e}\right) \alpha_{n} \frac{d_{0}}{e}-I\left(\alpha_{n} \lambda_{1}+\alpha_{n} \lambda_{2}\right) .
\end{aligned}
$$

The first-order participation constraint (foPC) is obtained by entering the optimal consumption values in (PC). We call it the first-order participation constraint since first-order approximations were used in the calculations of Proposition 4. The next step in the solution of the optimization problem for the intermediary is to replace (ISC) by a condition better suited to mathematical analysis. To find a manageable approximation of (ISC), while at the same time maintaining the main features, we first write the value function $V_{n}:=V\left(W_{n}, n,\left(f_{n}, T_{n+1}\right)^{\prime}\right)$ of an individual born at time $n$ as

$$
V_{n}=X_{n}+\Omega_{n} d_{1}
$$

where

and

$$
\Omega_{n}=\frac{1}{e}\left(u^{\prime}\left(\bar{c}_{n}\right) I^{\prime}(\bar{\lambda})+\beta u^{\prime}\left(\bar{c}_{n+1}\right) \mathbb{E}_{P}\left[I^{\prime}(\alpha \bar{\lambda})\right]\right)
$$

$$
\begin{aligned}
X_{n}=d_{0} \Omega_{n}+\bar{\lambda} \Omega_{n}-2\left(u^{\prime}\left(\bar{c}_{n}\right) I^{\prime}\left(\bar{c}_{n}\right)+\beta u^{\prime}\left(\bar{c}_{n+1}\right) \mathbb{E}_{P}\left[\bar{c}_{n+1}\right]\right) \\
+u\left(\bar{c}_{n}\right)+\beta u\left(\bar{c}_{n+1}\right) .
\end{aligned}
$$

Since value functions of two different groups of generations are compared in (ISC), we proceed in the following way to obtain an approximation. Let $z_{m} \equiv\left(W_{m}, m,\left(f_{m}, T_{m+1}\right)^{\prime}\right)$ and let $m=n+k$. Then

$$
\begin{aligned}
\sum_{m \in \Lambda_{2}} V\left(z_{m}\right) & =\sum_{n+k \in \Lambda_{2}} V\left(z_{n}+z_{n+k}-z_{n}\right) \\
& =\sum_{n+k \in \Lambda_{2}} V\left(z_{n}\right)+\left\langle\nabla V\left(y_{n}\right), y_{n+k}-y_{n}\right\rangle+o\left(\left\|y_{n+k}-y_{n}\right\|\right)
\end{aligned}
$$

and

$\sum_{n \in \Lambda_{1}} V\left(z_{n}\right)-\sum_{m \in \Lambda_{2}} V\left(z_{m}\right)=\sum_{n+k \in \Lambda_{2}}\left\langle\nabla V\left(y_{n}\right), y_{n+k}-y_{n}\right\rangle+o\left(\left\|y_{n+k}-y_{n}\right\|\right)$

using the fact that for fixed arguments the value function is invariant under time translation. For arbitrary $k$ and $\Lambda$ such that $|\Lambda|=\left|\Lambda_{1}\right|$, we obtain

$$
\sum_{n \in \Lambda_{1}} V\left(y_{n}\right)-\sum_{m \in \Lambda_{2}} V\left(y_{m}\right)=\sum_{n \in \Lambda}\left\langle\nabla V\left(y_{n}\right), y_{n+k}-y_{n}\right\rangle+o\left(\left\|y_{n+k}-y_{n}\right\|\right)
$$


implying the first-order intertemporal smoothing condition (foISC)

$$
\eta \geq\left|\sum_{n \in \Lambda}\left\langle\nabla V\left(W_{n}, n, f_{n}, T_{n+1}\right), W_{n, m}\right\rangle\right|
$$

for all $m=1,2, \ldots$ and

$$
\eta \geq\left|\sum_{n \in \Lambda} \Omega_{n}\left(f_{m, n}+T_{m, n}\right)\right|,
$$

where we have set $f_{m, n}:=f_{m}-f_{n}, T_{m, n}:=T_{m+1}-T_{n+1}$, and where $\Lambda$ denotes a subset of the natural numbers with cardinality $q>0$. The intermediary's optimization problem finally reads

$$
J\left(A_{0}\right)=\max _{\psi, y, f, T} \mathbb{E}\left[\sum_{n=0}^{\infty} \beta^{n} v\left(y_{n}\right)\right]
$$

subject to

$$
\begin{aligned}
& A_{n}=A_{n-1}+\left\langle\psi_{n-1}, \Delta S_{n}\right\rangle+f_{n}-T_{n}-y_{n}, A_{0} \text { given, } A_{n} \geq 0 \\
& \quad \eta \geq\left|\sum_{n \in \Lambda} \Omega_{n}\left(f_{m, n}+T_{m, n+1}\right)\right| \forall n \leq m, m=1,2, \ldots \\
& \frac{\gamma_{n}}{e} \mathbb{E}_{Q, n}\left[\frac{T_{n+1}}{B_{n+1}}\right] \geq \frac{\gamma_{n}}{e} f_{n}-\zeta_{n}+\tilde{\beta} \mathbb{E}_{Q, n}\left[\xi_{n}\right], \forall n \in \mathbb{N} .
\end{aligned}
$$

As a first step in the solution of (4.2), we consider the implications for the optimal risk-sharing contract while neglecting the optimal choice for $y_{n}$. Although (4.2) is a single agent optimization problem, it is not a standard problem since the number of constraints is infinite. This is due to the intertemporal smoothing constraint and the infinite number of individuals that the intermediary considers in the program.

Proposition 5. Assume that the technical conditions T.1-T.3 in Appendix $B$ hold. A necessary condition for an optimal risk-sharing contract $\left(f_{n}, T_{n+1}\right)^{\prime}$ to be offered by the intermediary at time $n$ is

$$
v_{f_{n}}^{\prime} \eta_{n} C_{n}=v_{n_{n}}^{\prime} C_{n}+\mathcal{A}_{n}
$$

with

$$
\begin{aligned}
\mathcal{A}_{n} & =\frac{\eta_{n}-1}{\beta_{n}} \frac{1}{q}\left(\left\langle v_{n}\right\rangle_{-n}-\left\langle v_{f}\right\rangle_{-n}\right) \\
C_{n} & =1-\frac{\eta_{n}-1}{\eta_{n}} \Omega_{n} q-\frac{\eta_{n}-1}{q \eta_{n}^{2}} \\
\left\langle v_{f}\right\rangle_{(-n)} & =\sum_{s=0, s \neq n}^{\infty} \frac{\beta^{s}}{\eta_{s}^{2}} v_{f_{s}}^{\prime} \\
\eta_{n} & =\mathbb{E}_{n}\left[\frac{L_{n+1} B_{n}}{L_{n} B_{n+1}}\right]
\end{aligned}
$$


where $v_{f}=\frac{\partial v}{\partial f}, v_{n}=\frac{\partial v}{\partial T}, v_{f_{n}}=\frac{\partial v}{\partial f_{n}}, v_{n_{n}}=\frac{\partial v}{\partial T_{n}}$.

Proof. Appendix B

From a structural point of view, (4.3) deviates from standard first-order conditions since the optimal choice of the contract components at any time $n$ depends not only on the respective marginal utilities, but also on the difference between the marginal utilities at all past and future times (the expression $\mathcal{A}_{n}$ ). $\mathcal{A}_{n}$ is therefore interpreted as a measure for the buffer stock used for intertemporal smoothing of consumption across the generations. A second unusual feature of (4.3) is the factor $\eta_{n}$ which weights the marginal utility of contract $f_{n}$. This factor reflects the costs the agents would face if the intermediary did not exist. If the financial markets are complete, $\eta_{n}=\frac{1}{B_{n+1}}$. The interaction of local and global features in the optimality condition (4.3) is due to the participation and the intertemporal smoothing condition.

To start with the economic implications of Proposition 5, we first consider $\mathcal{A}_{n}$. Let $\mathcal{A}_{n}<0$ or equivalently $\left\langle v_{n}\right\rangle_{(-n)}<\left\langle v_{f}\right\rangle_{(-n)}$. $\mathcal{A}_{n}<0$ can be interpreted as a growing buffer stock. Since $\eta_{n}$ and $C_{n}$ are both positive, it follows that $v_{f_{n}}^{\prime}<v_{n_{n}}^{\prime}$. Hence, if for each time $\mathrm{n}$ the averaged marginal utilities of the risky component of the contracts for all other generations are smaller than the corresponding average of the risk-free component $f_{\bullet}$, then the individual born at time $n$ will be compensated. On the other hand, if $\left\langle v_{n}\right\rangle_{(-n)}>\left\langle v_{f}\right\rangle_{(-n)}$, the individual born at time $n$ has to pay for the excess (expected) costs of the intermediary's payments to generations before and after $n$. This simple consideration highlights the interaction between generations.

We next consider the change in marginal utilities of the intermediary in (4.3) due to changes in the parameters.

$$
\frac{\partial v_{f_{n}}^{\prime}}{\partial \Omega_{n}}=\frac{1}{\eta_{n}} \mathcal{A}_{n} \frac{q}{C_{n}^{2} \eta_{n}} \begin{cases}>0 & \Leftrightarrow \mathcal{A}_{n}>0 \\ \leq 0 & \Leftrightarrow \mathcal{A}_{n}<0\end{cases}
$$

In order to interpret (4.7) we note that an increasing marginal utility $u^{\prime}$ of the individuals is a sufficient condition for $\Omega$ to increase. Therefore, whether a variation in the marginal utility of a single investor is positive or negative does not determine the impact of a variation on the intermediary's marginal utility - it is the aggregate over all past and future generations that determines the sign. This fact again captures the intuitive insight that if we link all generations in the intermediary's decision process, the single agent's characteristics are of little importance to the intermediary's performance. The impact of the market incompleteness measure $\eta_{n}$ is

$$
\frac{\partial v_{f_{n}}^{\prime}}{\partial \eta_{n}}=-\frac{1}{\eta_{n}^{2}} v_{n_{n}}^{\prime}-\mathcal{A}_{n} Z_{n}
$$


where

$$
Z_{n}=\frac{1}{\eta_{n}^{2}} \frac{1}{C_{n}}+\frac{1}{\eta_{n} C_{n}^{2}}\left(\frac{1}{\eta_{n}^{2}(1+q)}\right)>0
$$

This implies that for $\mathcal{A}_{n}>0, \frac{\partial v_{f_{n}}^{\prime}}{\partial \eta_{n}}<0$. An increase of $\eta_{n}$ might be due to a lower risk free interest rate and/or an increasing expected state price density. It thus becomes more attractive for the agents to enter into a risk-sharing contract. The increase in $\eta_{n}$ has a reverse impact on the behavior of the intermediary, which faces the same financial market as the agents. The net positive cash flow is reduced, as a decreased risk-free interest rate implies higher future contingent payments $T_{n}$. Therefore, for each additional dollar obtained at time $n-1$ in the risk-sharing contract, the intermediary expects to pay more to the individuals than in the case where $\eta_{n}$ is not increasing.

In the model considered so far, the individuals can voluntarily decide whether to accept or to reject the risk-sharing contract offered by the intermediary. In reality - especially in Europe - we observe compulsory membership of pension funds. Which system is superior is a notorious political issue. The compulsory membership model is presented in (2.1), but without the participation condition. If we let $\tilde{v}$ denote the utility function of the intermediary in the compulsory model, it follows from (B.1) and (B.2) in Appendix B that

$$
\tilde{v}_{f_{n}}^{\prime}=\tilde{v}_{n_{n}}^{\prime}
$$

is the optimality condition for the risk-sharing contract. Therefore, neither intertemporal smoothing nor the ratio between the state price densities and the risk-free interest rate matters. If we assume that $\tilde{v}_{n_{n}}^{\prime}=v_{n_{n}}^{\prime}$ holds, the optimality conditions imply

$$
\tilde{v}_{f_{n}}^{\prime}=v_{f_{n}}^{\prime} \eta_{n}-\frac{\mathcal{A}_{n}}{C_{n}} .
$$

Suppose $\mathcal{A}_{n}>0$, i.e., the buffer stock is increasing in size. Then

$$
\tilde{v}_{f_{n}}^{\prime}<v_{f_{n}}^{\prime} \eta_{n} \text {. }
$$

Since $\eta_{n}$ is decreasing - the denominator grows with $(1+r)^{n}$ and the nominator is bounded - the marginal utility for the intermediary $v_{f_{n}}^{\prime}$ has to increase in order to maintain the inequality (4.9). Hence, in the case $\mathcal{A}_{n}>0$, the intermediary is better off in the voluntary system. If $\mathcal{A}_{n}<0$, that is, where net payoffs are negative in the voluntary systems, it follows from condition 4.3 that $v_{f_{n}}^{\prime}$ is decreasing, i.e., the gap in the inequality $\tilde{v}_{f_{n}}^{\prime}>v_{f_{n}}^{\prime} \eta_{n}$ is increasing over time. The discussion shows that the question "Which pension fund system - voluntary or compulsory - is more efficient" is contingent on the performance of the financial markets. This is theoretical evidence for the observation that in the oil-shock period the German system outperformed the US system, while in the 90s booming stock markets made US residents better off (cf. Allen and Gale [1997] for a detailed discussion of the comparisons between Germany and the US). Since the performance of the stock markets is not known ex-ante, for a single pension fund the answer 
to the question of whether voluntary or compulsory membership is more efficient depends on the performance of equities. One solution, which would allow us to answer the question independently of stock market developments, is to consider more pension funds or security funds (fund-of-funds).

To illustrate some general results derived so far, we consider an example. We assume that all agents possess logarithmic utility of consumption and that the intermediary is characterized by a HARA-type utility $\frac{c^{p}-1}{p}, 0<$ $p<1$. Solving the participation constraint for the individuals born at time $n$ and $n+1$, we get for optimal consumption

$$
\begin{aligned}
c_{n}-\tilde{c}_{n} & =\frac{W_{n}}{1+\beta E_{Q, n}\left[\frac{L_{n+1}}{L_{n}}\right]}+\frac{E_{Q, n}\left[\frac{T_{n+1}}{B_{n+1}}\right]-f_{n}}{1+\beta E_{Q, n}\left[\frac{L_{n+1}}{L_{n}}\right]}=: M_{n, n+1} \\
c_{n+1}-\tilde{c}_{n+1} & =\beta B_{n+1} \frac{L_{n+1}}{L_{n}} M_{n, n+1}=: N_{n, n+1} M_{n, n+1} .
\end{aligned}
$$

It follows that the agents consume more (less) compared to the case without risk sharing if, and only if, the expected benefit of the contingent intermediary payment at retirement is larger than the deterministic payment when the agent is young. This result is in keeping with intuition. It follows from the expressions that the important qualitative factor - aside from the hitherto unspecified risk-sharing contracts - is the ratio of the state price densities at successive time periods. The next step is to apply these results in the intermediary's optimization problem. To simplify the calculations, we assume that the number of generations in the two pools $\Lambda_{1}, \Lambda_{2}$ is the same and equals $q$ and that the set $\Lambda_{2}$ is $k$ generations away from $\Lambda_{2}$. Therefore, for generation $n \in \Lambda_{1}$ there is a unique generation born at $n+k$ in $\Lambda_{2}$. Without loss of generality, we assume that in (ISC) the inequality

$$
\eta \geq \sum_{n=1}(1+\beta)\left(E_{Q, n}\left[N_{n, n+1}\right] M_{n, n+1}-E_{Q, k+n}\left[N_{k+n, k+n+1}\right] M_{k+n, k+n+1}\right)
$$

holds. In other words, an early generation in $\Lambda_{1}$ is expected to be more wealthy than a corresponding later one. If the assumption is reversed, all following statements are reversed too. It is then possible to derive sufficient optimality conditions for the intermediary. We recall that the choice variables are the two contract components and the investment strategy in the assets. We do not write the explicit optimality conditions, but merge the optimality conditions for $f_{n}$ and $f_{k+n}$, which eliminate the Lagrange multiplier of the ISC. We get 


$$
\begin{aligned}
& \underbrace{A_{n+1}-A_{n}+\left\langle\psi_{n-1}, \Delta S_{n}\right\rangle}_{\Delta_{n} \mathrm{M}-\mathrm{P}}+\underbrace{f_{n}-T_{n}}_{\Delta_{n} \mathrm{RS}-\mathrm{P}} \\
= & -\left(A_{n+k+1}-A_{n+k}+\left\langle\psi_{n+k-1}, \Delta S_{n+k}\right\rangle+f_{n+k}-T_{n+k}\right) \\
\times & \underbrace{\left(\frac{\beta}{1+\beta}\right)^{\frac{k}{p-1}}\left(\frac{1+\beta E_{Q, n}\left[\frac{L_{n+1}}{L_{n}}\right]}{1+\beta E_{Q, k+n}\left[\frac{L_{k+n+1}}{L_{k+n}}\right]}\right)^{\frac{1}{p-1}}}_{=: H_{n, n+k}} .
\end{aligned}
$$

We denote by $\Delta_{n}$ M-P ( $\Delta_{n}$ RS-P) the pure change in wealth of the intermediary due to the financial market $n$ (change in wealth due to the risksharing contract at time $n$ ). Hence, the optimality conditions enforce for the optimal contracts the following relationship between the agents in the different pools:

$$
\Delta_{n} \mathrm{M}-\mathrm{P}+\Delta_{n} \mathrm{RS}-\mathrm{P}=-\left(\Delta_{n+k} \mathrm{M}-\mathrm{P}-\Delta_{n+k} \mathrm{RS}-\mathrm{P}\right) \times H_{n, n+k} .
$$

This equation shows the essence of intertemporal smoothing: For different generations, the market risk and the longitudinal risk are equalized for the generations, which then defines the optimal risk-sharing contract. The "discount factor" $\mathrm{H}$ is based on the time preference rate of the intermediary, the temporal distance between the two pools of generations, and the state price density process. We only mention another characteristic of the optimality condition. Suppose that $k \rightarrow \infty$, i.e., the number of generations in each pool increases to infinity. Then the optimality condition for each generation $n$ is in the limit

$$
A_{n+1}-A_{n}+\left\langle\psi_{n-1}, \Delta S_{n}\right\rangle+f_{n}-T_{n}=0 .
$$

Hence, no risk sharing between the generations follows. A result that is in line with intuition.

We now discuss the full optimization problem (4.2) for the intermediary. Since we are interested in quantitative results too, we choose an explicit utility function $u$ given by

$$
u(y)=a_{0}+a_{1} y-\frac{1}{2} a_{2} y^{2}, a_{i}>0 \quad i=0,1,2 .
$$

The utility function is only meaningful for values of $y$ smaller than the satiating value. As a reminder, the no-arbitrage condition holds true, and we consider only self-financing strategies.

PROPOSITION 6. Consider problem (4.2) with utility function (4.11) for the intermediary. For the optimal shadow price of wealth it follows that

$$
\lambda=\frac{\sum_{k=0}^{\infty} \mathbb{E}_{Q}\left[\frac{a_{1} N_{k}}{a_{2} B_{k}}-\frac{f_{k}-T_{k}}{B_{k}}\right]-A_{0}}{\frac{1}{a_{2}} \mathbb{E}_{Q}\left[\sum_{k=0}^{\infty} \frac{N_{k}}{\beta^{k} B_{k}}\right]}
$$


with $N_{n}=\frac{\mathbb{E}_{P}\left[L \mid \mathcal{F}_{n}\right]}{B_{n}}$. Furthermore

$$
\frac{\partial \lambda}{\partial A_{0}}<0, \frac{\partial \lambda}{\partial f_{k}}<0, \frac{\partial \lambda}{\partial T_{k}}>0, \forall k \in \mathbb{N}
$$

If we assume that the (foISC) and the (foPC) do not bind, the optimal choice of $y$ is the satiating value $\hat{y}=\frac{a_{1}}{a_{2}}$.

Proof. Appendix C.

The inequalities in (4.12) are again in line with intuition. The fact that the optimal choice $y$ equals the satiating value $\frac{a_{1}}{a_{2}}$ if the constraints (foPC) and (foISC) are not binding can be interpreted as follows. With no limitations on the intermediary's debt capacity, there is a rational incentive to reduce the difference in value due to partial intertemporal smoothing and the willingness of the agents to enter into the risk-sharing contract with a nonbinding participation constraint. In other words, the intermediary uses the agent's willingness to enter into the contract as an infinite, deterministic income stream, and the possible risk-sharing repayment can either be financed as it arises given the intermediary's wealth, or the intermediary borrows the necessary amount. Therefore a limitation of the intermediary's ability to borrow $-A_{n}>0$ for example - is crucial for the individuals in a pension fund system. Otherwise, the possibility that the intermediary will be highly indebted is a source of large systemic risks.

The last issue we consider is the determination of the optimal risksharing contract component $T_{n+1}$. We assume that $T_{n+1}$ is a function of the risky assets $S_{n}^{(1)}$ and $S_{n}^{(2)}$. This implies that payments are contingent on the realization of the risky assets. Taking the derivatives of the optimal conditions with respect to $S_{n}^{(k)}$ and eliminating the Lagrange multipliers using the first-order conditions of the intermediary's problem $(2.1)^{1}$ we get

$$
\frac{\partial T}{\partial S_{n}^{(k)}}=\frac{\frac{u^{\prime} \phi_{n}^{(k)}}{u^{\prime \prime}}+\eta \psi_{n}^{(k)} \frac{v^{\prime \prime}}{v^{\prime}}}{\eta \frac{v^{\prime \prime}}{v^{\prime}}-(1-\eta) \frac{u^{\prime}}{u^{\prime \prime}}}, k=1,2 .
$$

\footnotetext{
${ }^{1}$ We use the full problem to analyze this question since the results are simpler than those where the constraints are replaced by their first-order approximations. In this case by taking the derivative of (B.1) and (B.2) in Appendix B with respect to the risky asset we get $S^{(k)}$ :

$$
(1-\eta)\left(\psi^{(k)}+\frac{1}{\eta} \frac{v^{\prime}}{v^{\prime \prime}} \frac{1}{C} \frac{\partial C}{\partial S^{(k)}}\right)=\frac{\partial T}{\partial S^{(k)}}, k=1,2 .
$$

Finally, using the expression for $C$ given in Equation (4.18) and calculating the derivative with respect to the risky asset implies

$$
\frac{\partial T}{\partial S_{n}^{(k)}}=\frac{\frac{u^{\prime}\left(c_{n}\right) I^{\prime} \phi_{n}^{(k)}}{\Omega}+\eta \psi_{n}^{(k)} \frac{v^{\prime \prime}}{v^{\prime}}}{\eta \frac{v^{\prime \prime}}{v^{\prime}}-(1-\eta) \frac{u^{\prime}\left(c_{n}\right)}{\Omega}}, k=1,2 .
$$
}


If we choose a specific utility function, (4.15) can be integrated and the optimal $T^{*}$ follows. As a result, for risk-neutral individuals and a riskneutral intermediary,

$$
\frac{\partial T}{\partial S_{n}^{(k)}}=0
$$

i.e., optimal payment is a constant and not a contingent claim. The optimal contract does not depend on any risk-neutrality assumption whatsoever.

$R_{I}$ and $R_{A}$ denote the Arrow-Pratt measures of risk aversion of the intermediary and of the individuals, respectively. For $\eta_{n}=1$,

$$
\frac{\partial T_{n}}{\partial S_{n}^{(k)}}=\psi_{n}^{(k)}+\phi_{n}^{(k)} \frac{1}{R_{A} R_{I}}, k=1,2
$$

and for $\eta_{n} \rightarrow 0$

$$
\frac{\partial T_{n}}{\partial S_{n}^{(k)}} \rightarrow-\phi_{n}^{(k)}, k=1,2 .
$$

Therefore, if the present value of the incomplete market measure vanishes, the slope of the optimal contract as a function of the asset prices is negative; the larger the risk exposure of the individual, the steeper the optimal contract. Note that $\eta_{n} \rightarrow 0$ for $n \rightarrow \infty$. Hence, in the long run the optimal contracting payment depends solely on the respective individual's risk exposure.

\section{Discussion}

Starting with the OLG model of Allen and Gale (1997), we extend it to cope with an intermediary that is also a utility maximizer. This extension allows us to analyze questions related to the optimal behavior of individuals and of an intermediary in an overlapping generational framework. The model implies that the initial wealth distribution of each generation is a crucial determinant of the individual participation at the intergenerational risksharing contract. It turns out that poor individuals have a stronger incentive to share risks across the generations than rich ones. We then show that as the individual time horizon increases, the importance of risk-sharing contracts decreases. Both results are in line with intuition, and the latter confirms the findings of Schechtman (1976) on self-insurance. The last finding regarding individual behavior was related to the feedback of a risk-sharing contract on the individual's investment strategy in the financial market. The risk exposure will therefore not increase for all individuals, but again the level of consumption determined by initial wealth and individual preferences determines the optimal risk exposure. Poor individuals will typically invest more in both risky assets, whereas the rich prefer to invest in the safe asset. Therefore, risk sharing has a positive impact on cross-sectional diversification for poor individuals. 
The optimization problem implied for the intermediary shows that the optimal risk-sharing contract equation possesses features which are not standard: For any point in time, the optimal contracts are not only functions of the individual born at the respective time, but also depend on the generations before and after the specific time. The first analysis of the optimality equations was done within the context of pension funds, in which we analyzed whether the intermediary is better off in a voluntary membership system or a compulsory one. The answer is contingent on the growth or decline of the buffer stock used to accomplish intertemporal smoothing of consumption over generations. If the reserves are growing, the voluntary system dominates. If the buffer stock is decreasing in size, the compulsory system is better for the intermediary. The last issue was a derivation of the optimal, explicit risk-sharing contracts as a function of the underlying stock price dynamics of the imperfect financial markets. An explicit system of differential equations was derived, which can be solved after specification of the preferences.

\section{Appendix A. Proof of Proposition 4}

Since we are interested in the optimal consumption paths, the martingale approach (see Pliska (1997) or Karatzas and Shreve (1998)) is used. We first consider the case in which it pays to accept the contract offered by the intermediary. By the martingale approach

$$
u\left(c_{n}-\bar{c}_{n}\right)+\beta \mathbb{E}_{n}\left[u\left(c_{n+1}-\bar{c}_{n+1}\right)\right]
$$

has to be maximized under the condition

$$
\left(c_{n}-\bar{c}_{n}\right)+\mathbb{E}_{Q, n}\left[\frac{1}{B_{n+1}}\left(c_{n+1}-\bar{c}_{n+1}-T_{n+1}\right)\right]=W_{n}-f_{n}
$$

where the expectation is taken with respect to the risk neutral probability $Q$. If we denote by $\lambda$ the Lagrange multiplier for (A.1), we obtain the necessary conditions

$$
\begin{aligned}
u^{\prime}\left(c_{n}-\bar{c}_{n}\right) & =\lambda \\
\beta u^{\prime}\left(c_{n+1}-\bar{c}_{n+1}\right) & =\lambda \frac{L_{n+1}}{B_{n+1} L_{n}} .
\end{aligned}
$$

The random variable $L_{n}=\frac{\mathrm{d} Q_{n}}{\mathrm{~d} P_{n}}$ is the so-called state price density, assumed to exist for all $n \in N . P_{n}$ and $Q_{n}$ denote the restrictions of $P$ and $Q$ on $\mathcal{F}_{n}$ respectively and are assumed to be locally equivalent (cf. Shiryaev (1999) for precise definitions). $L_{n}$ has to be introduced, since the expectation of the utility is taken with respect to the objective probability $P$, whereas condition (A.1) is written using the risk neutral probability $Q$. By assumption, $u$ is strictly increasing and strictly concave; therefore, $I=\left(u^{\prime}\right)^{-1}$ is well 
defined. (A.2) and (A.3) then imply for the optimal consumption of an individual born at time $n$

$$
\begin{aligned}
c_{n}^{*} & =I(\lambda)+\bar{c}_{n} \\
c_{n+1}^{*} & =I\left(\lambda \frac{L_{n}}{\beta B_{n+1} L_{n+1}}\right)+\bar{c}_{n+1} .
\end{aligned}
$$

Inserting (A.4) and (A.5) into (A.1) determines $\lambda$, i.e.

$$
I(\lambda)+\mathbb{E}_{Q, n}\left[\frac{1}{B_{n+1}}\left(I\left(\frac{\lambda L_{n}}{\beta B_{n+1} L_{n+1}}\right)-T_{n+1}\right)\right]=W_{n}-f_{n} .
$$

The implicit solution of the last equation is then reentered into (A.4) and (A.5) which finally yields the optimal consumption. The investment strategies can be calculated in a second step. To solve (A.6) at least approximately, we expand $I$ up to first order around $\bar{\lambda}$ :

$$
I(\lambda)=I(\bar{\lambda})+I^{\prime}(\bar{\lambda})(\lambda-\bar{\lambda})+o(|\lambda-\bar{\lambda}|)
$$

and

$$
I\left(\lambda \alpha_{n}\right)=I\left(\bar{\lambda} \alpha_{n}\right)+\alpha_{n}(\lambda-\bar{\lambda}) I^{\prime}\left(\bar{\lambda} \alpha_{n}\right)+o\left(\left|\alpha_{n}(\lambda-\bar{\lambda})\right|\right)
$$

in order to obtain (equalities are meant to hold up to first order)

$$
\lambda=\frac{d}{e}
$$

with

$$
\begin{aligned}
& d:= \underbrace{-\left(\bar{c}_{n}+\frac{\bar{c}_{n+1}}{B_{n+1}}\right)+I(\bar{\lambda})-\bar{\lambda} I^{\prime}(\bar{\lambda})-W_{n}+\mathbb{E}_{Q, n}\left[\frac{\alpha_{n}}{B_{n+1}}\left(\tilde{I}_{0}-I^{\prime}(\bar{\lambda})\right)\right]}_{d_{0}} \\
&+\underbrace{f_{n}-\mathbb{E}_{Q, n}\left[\frac{T_{n+1}}{B_{n+1}}\right]}_{d_{1}} \\
& e:=I^{\prime}(\bar{\lambda})+\mathbb{E}_{Q, n}\left[\frac{\alpha_{n}}{B_{n+1}}\left(\tilde{I}_{0}-I^{\prime}(\bar{\lambda})\right)\right], \alpha_{n}:=\frac{1}{\beta} \frac{L_{n+1}}{L_{n} B_{n+1}}, \tilde{I}_{0}(y)=I\left(\alpha_{n} y\right) .
\end{aligned}
$$

We next consider the individual in the case in which it pays for him not to accept the risk sharing contract. Then, the financial market is incomplete and we assumed that two risk neutral probabilities $Q_{1}$ and $Q_{2}$ in the closure of the set of risk neutral probabilities are a basis in the set of martingale measures. If we optimize the utility of the individual in this incomplete financial market using the martingale method, we have to consider two restrictions of the same type as (A.1), i.e.

$$
c_{n}-\bar{c}_{n}+\mathbb{E}_{Q_{i}, n}\left[\frac{1}{B_{n+1}}\left(c_{n+1}-\bar{c}_{n+1}\right)\right]=W_{n}, i=1,2 .
$$


Therefore two Lagrange multipliers $\lambda^{i}, i=1,2$ appear, but the analysis is identical and the expansion can be done around the same value as in the case of complete markets for both multipliers. We get

$$
\lambda^{1}=\frac{d_{2} e_{2}-d_{1} e_{4}}{e_{1} e_{4}-e_{2} e_{3}}, \lambda^{2}=\frac{d_{1} e_{3}-d_{2} e_{1}}{e_{1} e_{4}-e_{2} e_{3}}
$$

where

$$
\begin{gathered}
d_{i}=-\left(\bar{c}_{n}+\frac{\bar{c}_{n+1}}{B_{n+1}}\right)+I_{0}-I_{0}^{\prime} \bar{\lambda}-W_{n}+\mathbb{E}_{Q_{i}, n}\left[\frac{\alpha}{B_{n+1}}\left(\tilde{I}(\bar{\lambda})-I^{\prime}(\bar{\lambda})\right)\right], \\
e_{j}=I^{\prime}(\bar{\lambda})+\mathbb{E}_{Q_{1}, n}\left[\frac{\alpha_{n}^{(j)}}{B_{n+1}}\left(\tilde{I}(\bar{\lambda})-I^{\prime}(\bar{\lambda})\right)\right], j=1,2 \\
e_{k}=I^{\prime}(\bar{\lambda})+\mathbb{E}_{Q_{2}, n}\left[\frac{\alpha_{n}^{(k)}}{B_{n+1}}\left(\tilde{I}(\bar{\lambda})-I^{\prime}(\bar{\lambda})\right)\right], k=3,4
\end{gathered}
$$

and

$$
\alpha_{n}^{(1)}:=\frac{L_{n}^{(1)}}{\beta B_{n+1}} \quad \alpha_{n}^{(2)}:=\frac{L_{n}^{(2)}}{\beta B_{n+1}} .
$$

Inserting the Lagrange multipliers into the first order conditions determines the optimal consumption path $\left(c_{n}\right)$ while accepting the risk sharing contract and the path $\left(\tilde{c}_{n}\right)$ which is optimal in the incomplete financial market without the contract. Inserting the optimal Lagrange multipliers into the participation constraint (A.4) in Section 2 and expanding the constraint up to first order implies the formula given in Proposition 4.

\section{Appendix B. Proof of Proposition 5}

To prove Proposition 5, we first insert the budget constraint into the utility function of the intermediary, and then the first order conditions for the risk sharing contract are derived. Necessary conditions for the first order conditions to hold are

$$
\begin{aligned}
& 0=-\beta^{n} v_{f_{n}}^{\prime}+\lambda_{n} \frac{m}{e}+\sum_{s=0}^{\infty} \nu_{s} \Omega_{s}-\nu_{n} \Omega_{n} q, \forall \omega \\
& 0=\beta^{n} v_{n_{n}}^{\prime}+\mathbb{E}_{n}\left[\lambda_{n} \frac{N_{n}}{B_{n+1}}\right] \frac{m}{e}+\sum_{s=0}^{\infty} \nu_{s} \Omega_{s}-\nu_{n} \Omega_{n} q, \forall \omega,
\end{aligned}
$$

where $m$ is the shorthand notation for

$$
\gamma_{n, n+1}=I_{0}^{\prime}\left(\frac{d_{0}}{e}\right)-\tilde{\beta}_{n} \mathbb{E}_{Q, n}\left[I^{\prime}\left(\frac{\alpha d_{0}}{e}\right) \alpha\right]
$$

and $N_{n}=\frac{\mathbb{E}_{P}\left[L \mid \mathcal{F}_{n}\right]}{B_{n}}$. The sequence of Lagrange multipliers $\left(\lambda_{n}\right)$ is associated with (foPC) and the sequence $\left(\nu_{n}\right)$ with (foISC). Equations (B.1) and (B.2) 
have to hold for all states $\omega$ and they imply

$$
v_{f_{n}}^{\prime}=v_{n_{n}}^{\prime}+\mathbb{E}_{n}\left[\left(\frac{N_{n}}{B_{n+1}}-1\right)\right] \frac{\lambda_{n} m}{e \beta^{n}}, \forall \omega .
$$

Solving (B.1) with respect to $\lambda_{n}$ and inserting the result into (B.2) implies

$$
\mathcal{U}_{n}+\eta_{n}\left(\nu_{n} \Omega_{n} q-\sum_{s=0}^{\infty} \nu_{s} \Omega_{s}\right)=0, \forall \omega
$$

with $\mathcal{U}_{n}=\eta_{n} \beta^{n} v_{f_{n}}^{\prime}+\beta^{n} v_{n_{n}}^{\prime}$ and $\eta_{n}=\mathbb{E}_{n}\left[\frac{N_{n}}{B_{n+1}}\right]$. The last equation can be written for all $n$ as an infinite-dimensional linear system, i.e.

$$
\mathcal{U}=M \nu
$$

where $\mathcal{U}_{n}$ and $\nu_{n}$ are vectors $n=0,1,2, \ldots$ and $M$ is the matrix

$$
(M)_{i, j}=\left\{\begin{array}{ll}
x^{i} y^{i} & , i \neq j \\
a x^{i} b^{i} & , i=j
\end{array} \quad i, j=0,1,2, \ldots\right.
$$

where $x^{i}, y^{i}$ and $a$ are given by

\section{Assumption T.1.}

$$
x^{i}=\eta_{i}, y^{i}=\Omega_{i} \quad \text { and } \quad a=1-q .
$$

The intuition for this assumption can be gained from the explicit expressions for $\eta_{j}$ and $\Omega_{j}$. Since $\eta_{j}$ inhibits the growth dynamics of the riskless asset times the bounded measure of market incompleteness $\mathbb{E}\left[L \mid \mathcal{F}_{n}\right]$, the power law assumption is reasonable, and for $\Omega_{j}$ the power law assumption implies that we assume HARA-type utility functions for all the individuals. It is well known that this class of functions is large enough to contain various well known specific types of utility functions. The infinite dimensional linear system (B.5) is defined as the limit of finite dimensional systems where the matrices in each finite dimensional system have zero entries following an index $N$. We proceed as follows: First, we formally manipulate the system (B.5) and further operator equations below, and in a second step we define the mathematical setup which ex-post justifies the formal manipulations.

Assuming that the inverse operator $M^{-1}$ exists, we get

$$
\nu=M^{-1} \mathcal{U}
$$

for the sequence of Lagrange multipliers. Inserting this in the solution of (1) with respect to $\lambda_{n}$, i.e.

$$
\lambda=\left(\nu \Omega q+\beta v_{f}^{\prime}-\langle\nu, \Omega\rangle\right) \frac{e}{m}
$$

with

$$
\beta=\left(1, \beta, \beta^{2}, \ldots\right), v_{f}^{\prime}=\left(v_{f_{0}}^{\prime}, v_{f_{1}}^{\prime}, v_{f_{2}}^{\prime}, \ldots\right), \frac{e}{m}=\left(\frac{e_{0}}{\gamma_{0}}, \frac{e_{1}}{\gamma_{1}}, \frac{e_{2}}{\gamma_{2}}, \ldots\right)
$$


and $x y=\left(x_{1} y_{1}, x_{2} y_{2}, x_{3} y_{3}, \ldots\right)$, we get

$$
\lambda=\left(M^{-1} \mathcal{U} \Omega q+\beta v_{f}^{\prime}-\left\langle M^{-1} \mathcal{U}, \Omega\right\rangle\right) \frac{e}{m} .
$$

By passing to the first order conditions,

$$
\begin{aligned}
v_{f}^{\prime} & =v_{n}^{\prime}+\eta \lambda \frac{m}{e \beta}-\lambda \frac{m}{e \beta} \\
& =v_{n}^{\prime}+(\eta-1)\left(M^{-1} \mathcal{U} \Omega q+\beta v_{f}^{\prime}-\left\langle M^{-1} \mathcal{U}, \Omega\right\rangle\right) \frac{e}{m} \frac{m}{e \beta} \\
& =v_{n}^{\prime}+(\eta-1) v_{f}^{\prime}+\frac{(\eta-1)}{\beta} \mathcal{U} \Omega q-\frac{\eta-1}{\beta}\left\langle M^{-1} \mathcal{U}, \Omega\right\rangle .
\end{aligned}
$$

Replacing $\mathcal{U}$ by the explicit expression we finally get

$$
\begin{aligned}
v_{f}^{\prime}(\eta-(\eta-1) \Omega q)+ & \frac{\eta-1}{\beta}\left\langle M^{-1} \beta v_{f}^{\prime}, \Omega\right\rangle \\
& =v_{n}^{\prime}\left(1+\frac{\eta-1}{\eta} \Omega q\right)-\frac{\eta-1}{\beta}\left\langle M^{-1} \frac{\beta}{\eta} v_{n}^{\prime}, \Omega\right\rangle .
\end{aligned}
$$

We next analyze in which sense the infinite matrix $M$ and its inverse are well-defined. Therefore we first solve the system B.5 for a sub-system of dimension $N$, i.e. all entries in the vectors and the matrices are non-trivial for the first $N$ or $N \times N$ components and zero in the remaining components respectively. The operator $M$ is then a finite rank operator. The finite rank matrix $M^{N}$ is invertible and the inverse matrix is

$$
\left(M^{N,-1}\right)_{k l}= \begin{cases}\frac{N-3+a}{N-2+(N-2) a+a^{2}} \frac{1}{x^{k} y^{k}}=\frac{b_{N}}{a_{N}} \frac{1}{x^{k} y^{l}} & , k=l \\ \frac{1}{a_{N}} \frac{1}{x^{k} y^{l}} & , k \neq l\end{cases}
$$

$k, l=0,1,2, \ldots, N$. Given the finite rank expression for the inverse operator, we have to analyze first in which sense the infinite dimensional operator $M^{-1}$ is well-defined and second, what is an explicit expression for this operator. Therefore, we first determine the conditions such that $M^{-1}$ is a trace class operator and note that

$$
\begin{aligned}
A_{N} & =\sum_{k, l=0}^{N}\left|\gamma_{k l}^{-1}\right|=\sum_{k=l}^{N}\left|\gamma_{k l}^{-1}\right|+\sum_{k \neq l}^{N}\left|\gamma_{k l}^{-1}\right| \\
& =\frac{b_{N}}{a_{N}} \frac{1-\left(\frac{1}{x y}\right)^{N+1}}{1-\frac{1}{x y}}+\frac{1}{a_{N}}\left(\frac{1-\left(\frac{1}{x}\right)^{N+1}}{1-\frac{1}{x}} \frac{1-\left(\frac{1}{y}\right)^{N+1}}{1-\frac{1}{y}}-\frac{1-\left(\frac{1}{x y}\right)^{N+1}}{1-\frac{1}{x y}}\right) .
\end{aligned}
$$

AsSUMPTION T.2. If we assume $\left|\frac{1}{x}\right|<1,\left|\frac{1}{y}\right|<1$ and all vectors are square summable, then the limit

$$
\lim _{N \rightarrow \infty} A_{N}=\frac{1}{1+a} \frac{1}{1-\frac{1}{x y}}
$$


exists and the operator $M^{-1}$ is a trace class operator (see Gohberg et al. (1990) for properties of trace class operators).

Given this setup, we can calculate the scalar products in (B.7):

$$
\begin{aligned}
\left\langle M^{-1} \beta v_{f}^{\prime}, \Omega\right\rangle & =\sum_{s=0}^{\infty} \sum_{j=0}^{\infty} m_{s j}^{-1} \beta^{j} v_{f_{j}}^{\prime} \Omega_{s} \delta_{s j} \\
& =\sum_{s=0}^{\infty} m_{s s}^{-1} \beta^{s} v_{f_{s}}^{\prime} \Omega_{s}=\frac{1}{q} \sum_{s=0}^{\infty}\left(\frac{\beta}{x}\right)^{s} v_{f_{s}}^{\prime} .
\end{aligned}
$$

If we finally assume the growth condition for the marginal utilities

AsSUMPTION T.3.

$$
\left|v_{f_{s}}^{\prime}\right| \leq \text { const },\left|v_{n_{s}}^{\prime}\right| \leq c^{s}, c>0, \forall s,\left|\frac{\beta c}{x}\right|<1,
$$

then $\frac{1}{q} \sum_{s=0}^{\infty}\left(\frac{\beta}{x}\right)^{s} v_{f_{s}}^{\prime}$ converges.

Using this assumption, we decompose the scalar products into a time $n$-term and the remaining ones, i.e.

$$
\begin{aligned}
\left\langle M^{-1} \beta v_{f}^{\prime}, \Omega\right\rangle & =\frac{1}{q} \sum_{s=0, s \neq n}^{\infty}\left(\frac{\beta}{x}\right)^{s} v_{f_{s}}^{\prime}+\frac{1}{q}\left(\frac{\beta}{x}\right)^{n} v_{f_{n}}^{\prime} \\
& =\frac{1}{q}\left\langle v_{f}\right\rangle_{(-n)}+\frac{1}{q}\left(\frac{\beta}{x}\right)^{n} v_{f_{n}}^{\prime} \\
\left\langle M^{-1} \frac{\beta}{\eta} v_{n}^{\prime}, \Omega\right\rangle & =\frac{1}{q} \sum_{s=0, s \neq n}^{\infty}\left(\frac{\beta}{x^{2}}\right)^{s} v_{f_{s}}^{\prime}+\frac{1}{q}\left(\frac{\beta}{x^{2}}\right)^{n} v_{f_{n}}^{\prime} \\
& =\frac{1}{q}\left\langle v_{n}\right\rangle_{-n}+\frac{1}{q}\left(\frac{\beta}{x^{2}}\right)^{n} v_{f_{n}}^{\prime} .
\end{aligned}
$$

Inserting (B.12) and (B.13) into (B.8) implies after some algebra

$$
\begin{gathered}
v_{f_{n}}^{\prime} \eta_{n} C_{n}=v_{n_{n}}^{\prime} C_{n}+\mathcal{A}_{n} \\
\mathcal{A}_{n}=\frac{\eta_{n}-1}{\beta_{n}} \frac{1}{q}\left(\left\langle v_{n}\right\rangle_{-n}-\left\langle v_{f}\right\rangle_{-n}\right) \\
C_{n}=1-\frac{\eta_{n}-1}{\eta_{n}} \Omega_{n} q-\frac{\eta_{n}-1}{q \eta_{n}^{2}} .
\end{gathered}
$$

This proves Proposition 5.

\section{Appendix C. Proof of Proposition 6}

The first step in the proof is to rewrite the budget constraint in a suitable form in order to apply the martingale approach. We write

$$
\frac{A_{n+1}}{B_{n+1}}=A_{0}+G_{n+1}^{*}-\sum_{k=0}^{t} \frac{y_{n}-T_{k}+f_{k}}{B_{k}}
$$


with

$$
G_{n}^{*}=\sum_{s=1}^{t} \sum_{n=1}^{2} \psi_{n}(s) S^{*}(s)
$$

being the gain process and $S^{*}(n)=\frac{S(n)}{B(n)}$ the normalized prices. Since $A_{n}=$ $A_{0}+G_{n}^{*}$ is a $Q$-martingale and since we are interested only in self-financing strategies, it follows that (see Pliska (1997), Chapter V, for example)

$$
A_{0}=\mathbb{E}_{Q}\left[\sum_{k=0}^{\infty} \frac{y_{n}-T_{k}+f_{k}}{B_{k}}\right] \text {. }
$$

ASSUMPTION T.4. We assume that the integral

$$
\mathbb{E}_{Q}\left[\sum_{k=0}^{\infty} \frac{y_{n}-T_{k}+f_{k}}{B_{k}}\right]
$$

converges in the $L^{2}$ norm with $n \rightarrow \infty$.

In the problem (4.4) we replace the budget constraint by (C.2) while the objective function, the (foISC) and the (foPC) remain unchanged. A sufficient condition for the first order condition for the intermediary with the quadratic utility function is

$$
a_{1}-a_{2} y_{n}=\beta^{-t} \lambda \frac{N_{n}}{B_{n}}
$$

with $N_{n}=\frac{\mathbb{E}_{P}[L \mid\{n]}{B_{n}}$ and $\lambda$ the Lagrange multiplies associated with (C.2). Solving (C.3) with respect to $y_{n}$ and inserting the solution into (C.2) determines the Lagrange multiplier

$$
\lambda=\frac{\sum_{k=0}^{\infty} \mathbb{E}_{Q}\left[\frac{a_{1} N_{k}}{a_{2} B_{k}}-\frac{f_{k}-T_{k}}{B_{k}}\right]-A_{0}}{\frac{1}{a_{2}} \mathbb{E}_{Q}\left[\sum_{k=0}^{\infty} \frac{N_{k}}{\beta^{k} B_{k}}\right]}
$$

where again the integrals are assumed to converge in the $L^{2}$ sense. The derivatives in Proposition 7 trivially follow from (C.3) and (C.4). If we assume that (foISC) and (foPC) do not bind, then by slackness, the sequences of Lagrange multipliers for these conditions are equal to 0 , which in turn implies $\lambda=0$. But then the optimal choice is $\hat{y}=\frac{a_{1}}{a_{2}}$.

\section{References}

Allen, F., and Gale, D. (1997) Financial Markets, Intermediaries and Intertemporal Smoothing, J. Pol. Econom., 105, 523-546.

Bodie, Z., Merton R. C., and Samuelson W. (1992) Labor Supply Flexibility and Portfolio Choice in a Life Cycle Model, J. Econom. Dynam. Control, 16, 427-450.

Davis, E. (1995) Pension Funds: Retirement-Income Security and Capital Markets, Oxford University Press, New York. 
Gohberg, I., Goldberg S., and Kaashoeck M. (1990) Classes of Linear Operators, Vol. I, Operator Theory: Advances and Applications in Mathematical Finance, IMA Vol. 65. Birkhäuser Verlag, Basel.

Hernandez-Lerma, O., and Lassere J. (1996) Basic Optimality Criteria, Applications of Mathematics, Stochastic Modeling and Applied Probability, in Discrete-Time Markov Control Processes, ed. by I. Karatzas, and M. Yor, Vol. 30. Springer, New York.

Jorion, P. (2000) The Long-Term Risks of Global Stock Markets, Working Paper, University of California at Irvine.

Karatzas, I., and Shreve S. E. (1998) Methods of Mathematical Finance, Springer Verlag.

Pliska, S. R. (1997) Introduction to Mathematical Finance: Discrete Time Models, Blackwell Publishers.

Schechtman, J. (1976) An Income Fluctuation Problem, J. Econom. Theory, 12, 218-241.

Shiryaev, A. N. (1999) Essentials of Stochastic Finance: Facts, Models, Theory, Vol. 3, Advanced Series on Statistical Science and Applied Probability, World Scientific, Singapore 


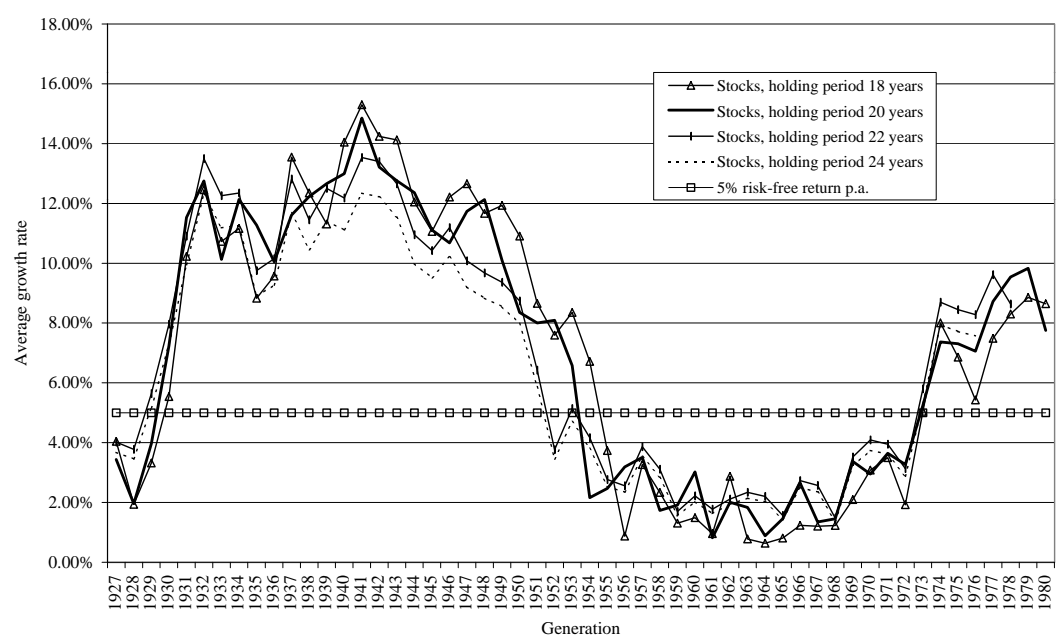

FIGURE 1. The figure shows the average growth rate for a holding period of 18, 20, 22 and 24 years. Each generation invests either in the risk free asset (assumed to yield 5 percent p.a.) or in the risky asset. The risky asset return is the value-weighted return on all NYSE, AMEX, and NASDAQ stocks. Source of data: Kenneth R. French webpage, http://mba.tuck.dartmouth.edu/pages / faculty/ken.french/datalibrary.html. 

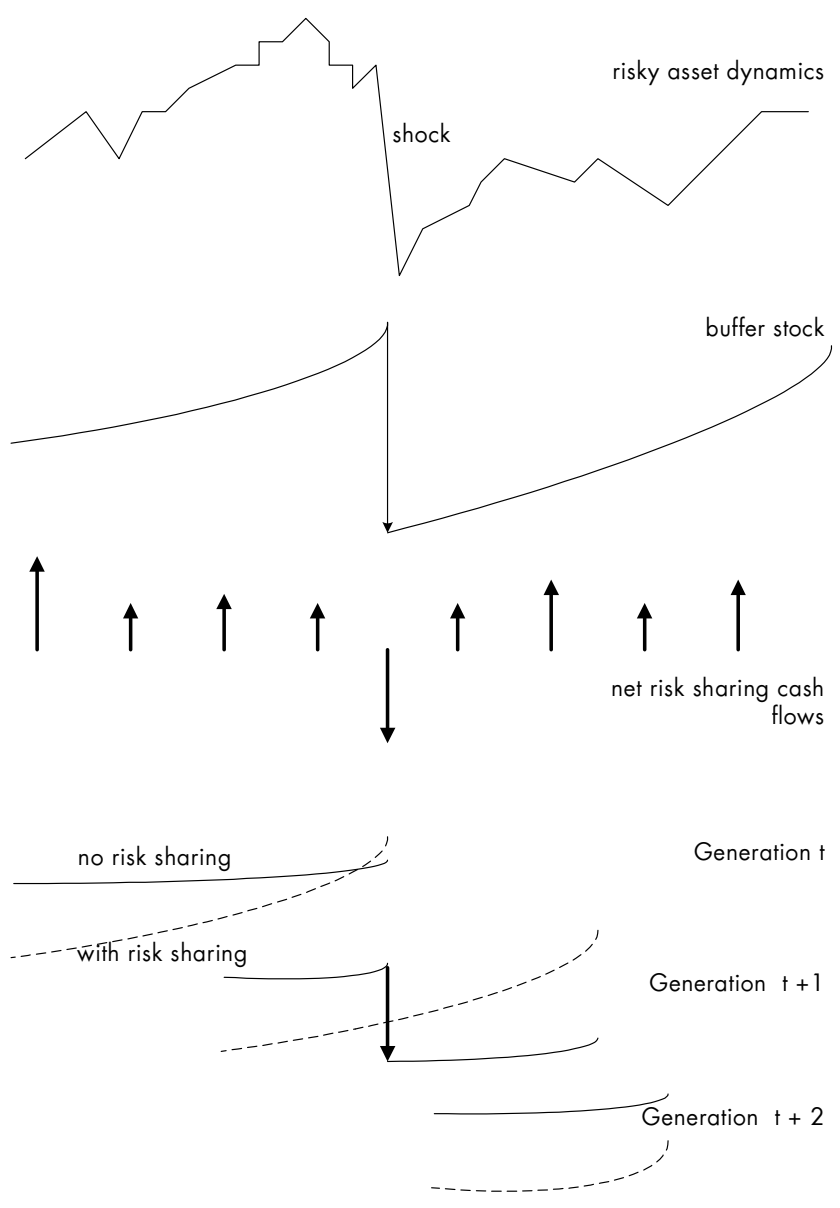

FIGURE 2. The figure illustrates the risk sharing mechanism across the generations accomplished by an intermediary. Three representative individuals of three different generations are shown. Generation 2 is hit by a shock and the figure shows the losses in life time consumption for the individual of generation 2 without a risk-sharing contract (dashed lines). In contrast, the consumption path with risk-sharing contracts is shown. The smoothing of consumption is due to the buffer stock, i.e. contributions of generations earlier than 2 and expected contributions of generations after 2 are used to smooth consumption of the unlucky generation 2 . 


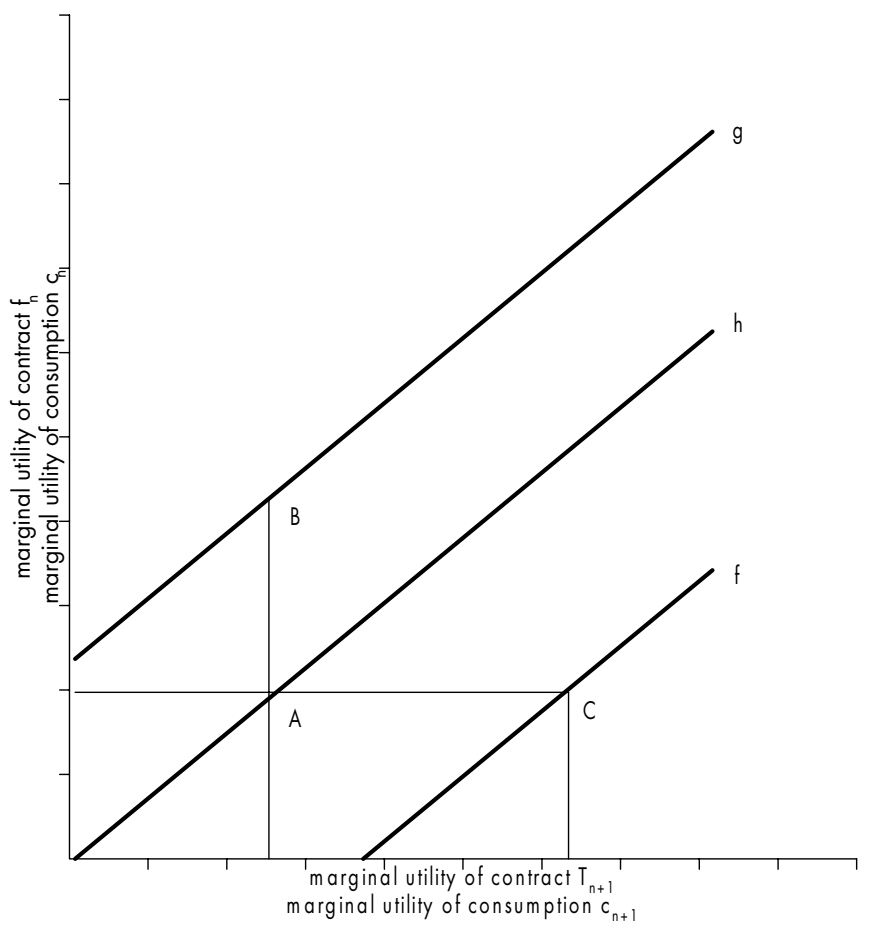

FIGURE 3

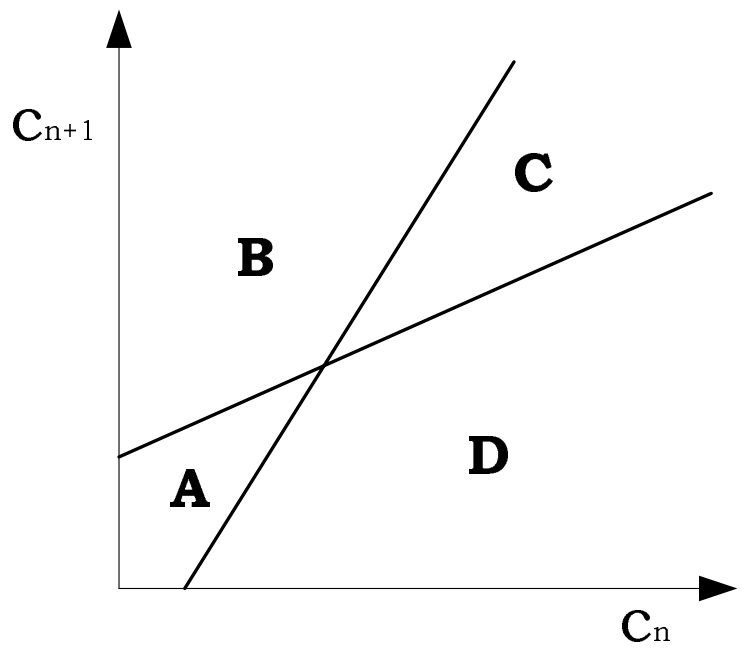

FIGURE 4. Today's consumption $c_{n}$ and tomorrow's consumption $c_{n+1}$ of an individual born at time $n$ are displayed on the axis. The four regions $A, B, C, D$ represent different investments into the two risky assets $S_{n}^{(1)}$ and $S_{n}^{(2)}$ of the individual if he accepts the offered risk sharing contract relative to the case without such a contract. 